\title{
Temperature-insensitive flexible polymer wavelength filter fabricated on polymer substrates
}

\author{
Sung-Hyun Nam \\ Information and Electronic Materials Research Institute, LG Chemical, Limited, Research Park, \\ Yuseong-gu, Daejeon 305-380, Korea \\ Jae-Wook Kang and Jang-Joo Kim ${ }^{\mathrm{a})}$ \\ School of Materials Science and Engineering and OLED Center, Seoul National University, \\ Seoul 151-744, Korea
}

(Received 9 August 2005; accepted 12 October 2005; published online 30 November 2005)

\begin{abstract}
Temperature-insensitive polymer wavelength filters with silicon nitride gratings were fabricated on a polymer substrate. Polymer waveguides on polymer substrates were fabricated on top of the $\mathrm{Si}$ wafer with a water soluble poly(vinylalcohol) (PVA) interlayer between the Si wafer and polymer waveguides. After the completion of the waveguides, the polymer waveguides are separated from the substrate by dissolving the PVA layer to form all polymer waveguides. The novel process provides a way to overcome the problem of the thermal instability of polymer substrate when the polymer waveguides are directly fabricated on polymer substrates. The polymeric wavelength filter fabricated on a polymer substrate exhibits one order of magnitude lower Bragg wavelength shift with temperature than the device on the $\mathrm{Si}$-wafer in the temperature range of $25-70{ }^{\circ} \mathrm{C}$, which is consistent with theoretical prediction. (C) 2005 American Institute of Physics.
\end{abstract}

[DOI: $10.1063 / 1.2140469]$

Wavelength filters are essential components for the optical communication systems based on wavelength division multiplexing (WDM) in order to select one wavelength carrying a desired signal. ${ }^{1,2}$ However the temperature dependence of the central wavelength of the reflected signal in the filters is a serious and outstanding problem for WDM systems. It is mostly caused by the temperature dependence of optical path length of devices. ${ }^{3-7}$ Since this temperature sensitivity causes the crosstalk in the dense WDM systems with very small channel spacing, such narrow-band optical filters have required a precise temperature control unit. These problems can be solved by fabricating athermal (temperature-

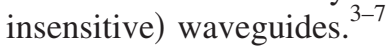

The Bragg wavelength, $\lambda_{0}$ is expressed as follows:

$$
\lambda_{0}=2 \Lambda_{g} N_{\text {eff }},
$$

where $N_{\text {eff }}$ is the effective refractive index of the guided mode in the filter and $\Lambda_{g}$ is the grating period. ${ }^{2}$ The temperature dependence of the central wavelength of filters is expressed by $^{8}$

$$
\begin{aligned}
\frac{d \lambda_{0}}{d T} & =2\left(N_{\mathrm{eff}} \frac{d \Lambda_{g}}{d T}+\Lambda_{g} \frac{d N_{\mathrm{eff}}}{d T}\right) \\
& =2 \Lambda_{g}\left(N_{\mathrm{eff}} \frac{1}{\Lambda_{g}} \frac{d \Lambda_{g}}{d T}+\frac{d N_{\mathrm{eff}}}{d T}\right) .
\end{aligned}
$$

Thus, $d \lambda_{0} / d T$ is given by the following equation:

$$
\frac{d \lambda_{0}}{d T}=2 \Lambda_{g}\left(N_{\mathrm{eff}} \alpha_{\mathrm{sub}}+\frac{d N_{\mathrm{eff}}}{d T}\right),
$$

where $d N_{\text {eff }} / d T$ is the thermo-optic (TO) coefficient of the waveguide, and $\alpha_{\text {sub }}\left(=1 / \Lambda_{g} \cdot d \Lambda_{g} / d T\right)$ is the coefficient of

\footnotetext{
a) Author to whom correspondence should be addressed; electronic mail: jjkim@snu.ac.kr
}

thermal expansion (CTE) of the substrate. Since the substrate is usually much thicker than that of the waveguide layer, the coefficient of thermal expansion is determined by the substrate. ${ }^{3}$ The athermal wavelength filter which shows no temperature dependence of the central wavelength of the reflected light can be realized by properly selecting the substrate and waveguide material to satisfy the following condition:

$$
N_{\mathrm{eff}} \alpha_{\mathrm{sub}}=\frac{d N_{\mathrm{eff}}}{d T}=0 .
$$

One needs to notice that it is not possible to realize athermal filters using silica based waveguides because $\alpha_{\text {sub }} \quad\left(2.63 \mathrm{ppm}\right.$ for silicon wafer) and $d N_{\text {eff }} / d T$ $\left(\approx+10^{-6}{ }^{\circ} \mathrm{C}^{-1}\right.$ for silica $)$ are both positive so that $d \lambda_{0} / d T$ is always positive. In contrast, polymer waveguides allow achieving athermal devices by choosing a suitable combination of substrate and waveguide material with a negative TO value.

One difficulty of the fabrication of polymer waveguides on polymer substrates is the thermal instability of the substrates. Polymer waveguides are formed by spin coating of polymer solution followed by baking at elevated temperature to dry out the solvent. The baking process induces bending and shrinking of the substrates to make the process almost impossible.

In this paper, we report on temperature-insensitive wavelength filters using a new process to fabricate the devices on a polymer substrate. In the process, polymer waveguides are fabricated on a silicon wafer with a water soluble interlayer, poly(vinylalcohol) (PVA), between the waveguides and the substrate. A thick polymer layer, which was deposited on top of the waveguides by the doctor blade technique, is used as the substrate. Dissolving out the interlayer completes the polymer waveguide devices on the polymer substrate. The 


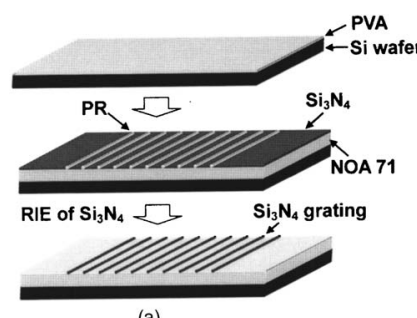

(a)

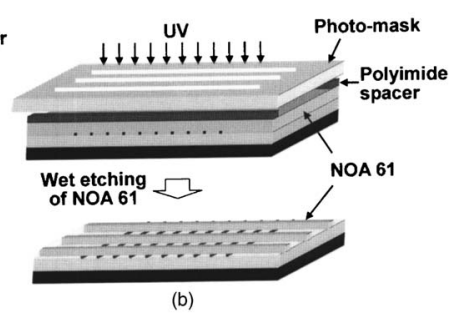

(b)

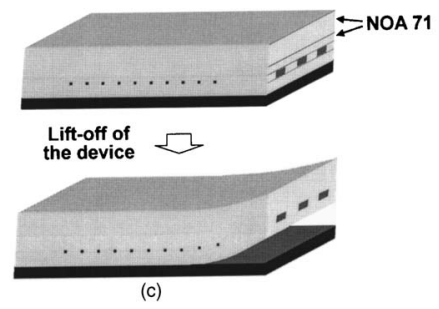

FIG. 1. Schematic diagram of the flexible wavelength filter on polymer substrate. (a) The transfer of PR grating as an etching mask to the $\mathrm{Si}_{3} \mathrm{~N}_{4}$ layer by $\mathrm{O}_{2}$ and $\mathrm{CF}_{4} \mathrm{RIE}$, (b) the fabrication of channel waveguide using the proximate-contact lithography, and (c) lift-off of the device from Si wafer by dissolving the PVA layer in DI water after spin-coating of the NOA71 as the upper cladding and substrate.

process was applied to fabricate a temperature-insensitive wavelength filter.

Figure 1 shows the schematic diagram of the fabrication of the flexible polymer wavelength filter on a polymer substrate. UV-curable resins, NOA61 (Norland Products Inc. and $n^{\mathrm{TE}}=1.547$ at $1550 \mathrm{~nm}$ wavelength) and NOA71 (Norland Products Inc. and $n^{\mathrm{TE}}=1.540$ at $1550 \mathrm{~nm}$ wavelength), were used as the core and cladding materials, respectively. NOA71 was also used as the polymer substrate. After successive spin-coating and baking of $15-\mu \mathrm{m}$-thick PVA and NOA71 on the $\mathrm{Si}$ wafer, a 50-nm-thick silicon nitride $\left(\mathrm{Si}_{3} \mathrm{~N}_{4}\right)$ layer was deposited by plasma enhanced chemical vapor deposition. The Bragg gratings were fabricated by the holographic interference method using a photoresist (PR) and $\mathrm{Ar}^{+}$laser of $488 \mathrm{~nm}$ wavelength. ${ }^{9}$ The PR gratings were transferred onto the $\mathrm{Si}_{3} \mathrm{~N}_{4}$ layer $(n=1.8)$ by using $\mathrm{O}_{2}$ and $\mathrm{CF}_{4}$ reactive ion etching (RIE) to induce a large difference of effective index between the grating layer and the core layer of the waveguide [Fig. 1(a)]. The period and depth of the transferred gratings were $495 \mathrm{~nm}$ and $50 \mathrm{~nm}$, respectively. 3.5- $\mu$ m-thick NOA61 layer was spin coated on the $\mathrm{Si}_{3} \mathrm{~N}_{4}$ grating and the waveguide patterns were formed by proximate-contact lithography using negative-photomask attached to $25-\mu \mathrm{m}$-thick polyimide spacers followed by wet chemical etching of the unexposed area [Fig. 1(b)]. ${ }^{10,11}$ After spin coating and curing of the upper cladding layer (NOA71, $10 \mu \mathrm{m}$ thick), 1.5 -mm-thick NOA71 was coated as the polymer substrate by the doctor blade technique on the device. The NOA71 substrate was cured by UV with the intensity of $100 \mathrm{~mW} / \mathrm{cm}^{2}$ for $25 \mathrm{~min}$. Separation of the device from Siwafer by dissolving the PVA layer in DI water completes the process [Fig. 1(c)].

Figure 2 shows waveguide patterns fabricated on the $\mathrm{Si}_{3} \mathrm{~N}_{4}$ grating and the picture of the fabricated waveguide devices. The grating has a uniform period of $495 \mathrm{~nm}$ and a depth of $50 \mathrm{~nm}$. These results indicate that the grating has been successfully fabricated by the process. The waveguide

is well defined and has smooth side walls.
Downloaded 31 Jan 2006 to 147.46 .94 .102 . Redistribution subject to AlP license or copyright, see http://apl.aip.org/apl/copyright.jsp
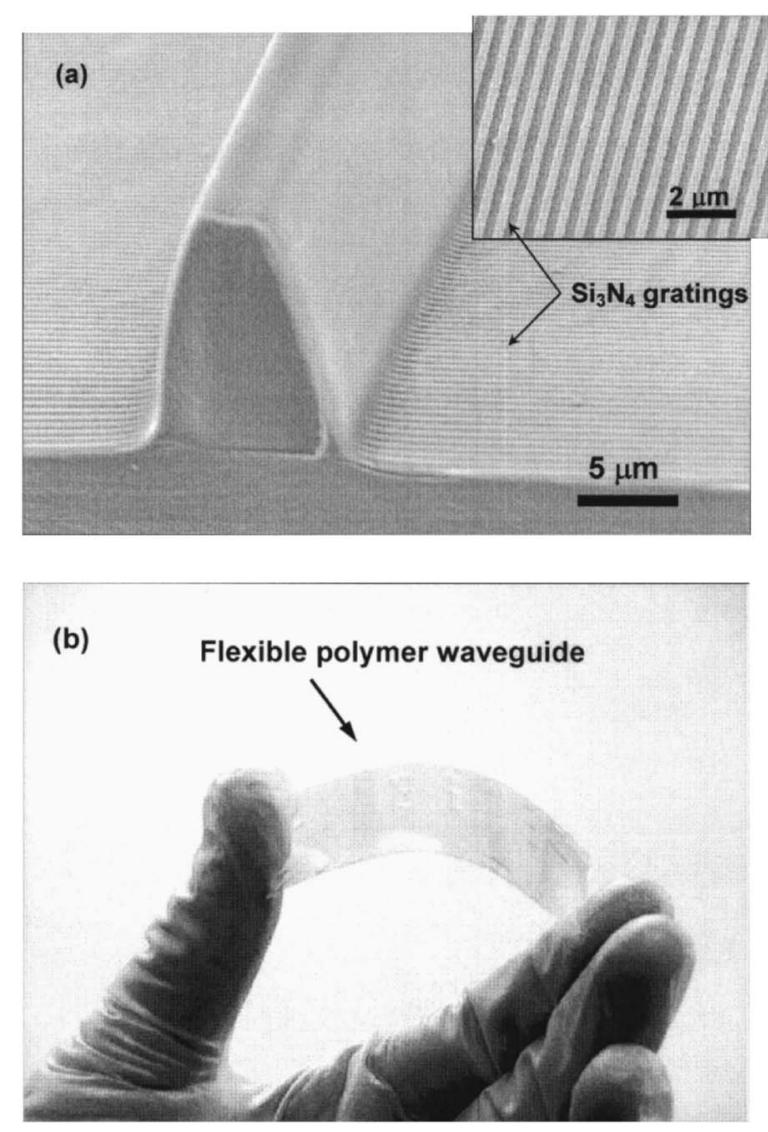

FIG. 2. (a) SEM image of the channel waveguide pattern fabricated by proximate-contact lithography on $\mathrm{Si}_{3} \mathrm{~N}_{4}$ gratings defined by $\mathrm{O}_{2}$ and $\mathrm{CF}_{4}$ RIE, and (b) picture of the flexible wavelength filter fabricated on polymer substrate.

In order to measure the Bragg reflections of the device, a tunable laser source (HP81642A) operating around $1550 \mathrm{~nm}$ or broadband light source (HP83437A) covering the wavelength from $1100 \mathrm{~nm}$ to $1700 \mathrm{~nm}$ was used as an input light source through the single mode fiber. The output of the device was also launched to a single mode fiber and the output light was monitored with an optical power sensor (HP81635A) or an optical spectrum analyzer (HP86140B). The length of the device containing 10-mm-long Bragg grating was $3 \mathrm{~cm}$. The polarization of the input light was adjusted to TE polarization using a polarization controller throughout the measurement.

The temperature dependence of the Bragg wavelength $\left(d \lambda_{0} / d T\right)$ was measured in the temperature range between 25 and $70{ }^{\circ} \mathrm{C}$ by placing the waveguide on a temperature controllable waveguide holder (Suruga Seiki F274-18). Figures 3(a) and 3(b) exhibit the shifts of the transmission spectra of the optical filter when the temperature was increased from 25 to $70{ }^{\circ} \mathrm{C}$, and the temperature dependence of the Bragg wavelength of the filters with the temperature sensitivity (slope) of $-0.021 \mathrm{~nm} /{ }^{\circ} \mathrm{C}$, respectively.

The experimentally obtained $d \lambda_{0} / d T$ was compared with theoretically estimated values using the Eq. (4). We measured TO coefficient of NOA61 at the wavelength of $1550 \mathrm{~nm}$ by using the prism coupler with a hot stage to control the temperature of the film. The measured TO coefficient $(d n / d T)$ was $-2.46 \times 10^{-4}{ }^{\circ} \mathrm{C}^{-1}$ and the calculated $d N_{\text {eff }} / d T$ using the beam propagation software (BeamPROPTM, RSoft Design Group) was $-2.38 \times 10^{-4}{ }^{\circ} \mathrm{C}^{-1}$ for TE polarization at 

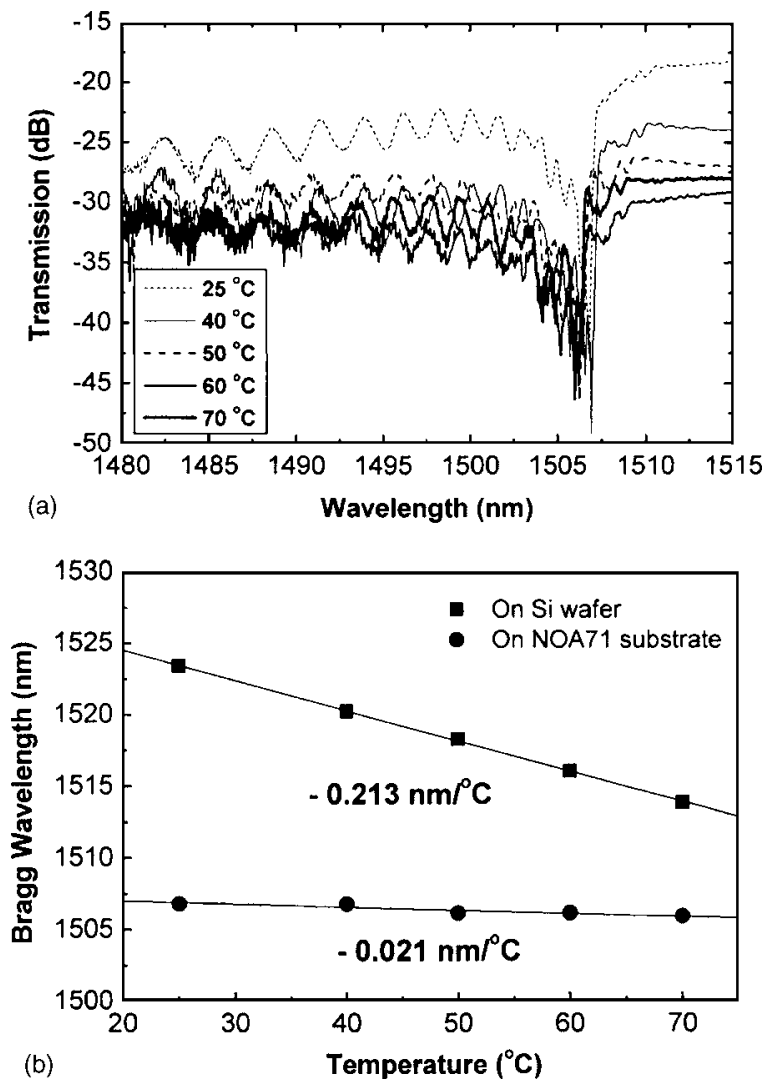

FIG. 3. (a) Transmission spectra of the Bragg grating filter fabricated on NOA71 substrate at different temperature, and (b) temperature dependence of the Bragg wavelength of the filter fabricated on Si wafer and NOA71 substrate, respectively.

$1550 \mathrm{~nm}$ wavelength. The CTE value of NOA71 substrate was measured by TMA (Thermal Mechanical Analyzer, SEIKO TMA120). Figure 4 shows the displacement changes of NOA71 material with temperature in TMA measurements. The dimension of the sample for the measurement was $10 \times 10 \times 1.5 \mathrm{~mm}^{3}$. The sample was measured with the heating rate of $5{ }^{\circ} \mathrm{C} / \mathrm{min}$ and the load of $20 \mathrm{~g}$. The measured CTE value of the NOA71 polymer from the slope in the figure was $142.5 \mathrm{ppm} /{ }^{\circ} \mathrm{C}$. The effective refractive index of $6 \times 3.5 \mu \mathrm{m}^{2}$ (width $\times$ height) waveguide with 50 -nm-thick $\mathrm{Si}_{3} \mathrm{~N}_{4}$ gratings is 1.5422 calculated using BeamPROPTM. Therefore, the calculated temperature dependence of the wavelength shift $\left(d \lambda_{0} / d T\right)$ of the polymer wavelength filter is $-0.018 \mathrm{~nm} /{ }^{\circ} \mathrm{C}$, which is in good agreement with the experimental result of $-0.021 \mathrm{~nm} /{ }^{\circ} \mathrm{C}$.

The temperature dependence of the Bragg wavelength of the filters fabricated on the Si-wafer is compared with the device on the polymer substrate in Fig. 3(b). The device exhibits a large shift of the Bragg wavelength from 1524 to $1514 \mathrm{~nm}$ as the temperature increases from 25 to $70{ }^{\circ} \mathrm{C}$ with the temperature sensitivity of $-0.213 \mathrm{~nm} /{ }^{\circ} \mathrm{C}$. The temperature sensitivity of the Bragg wavelength was reduced by one

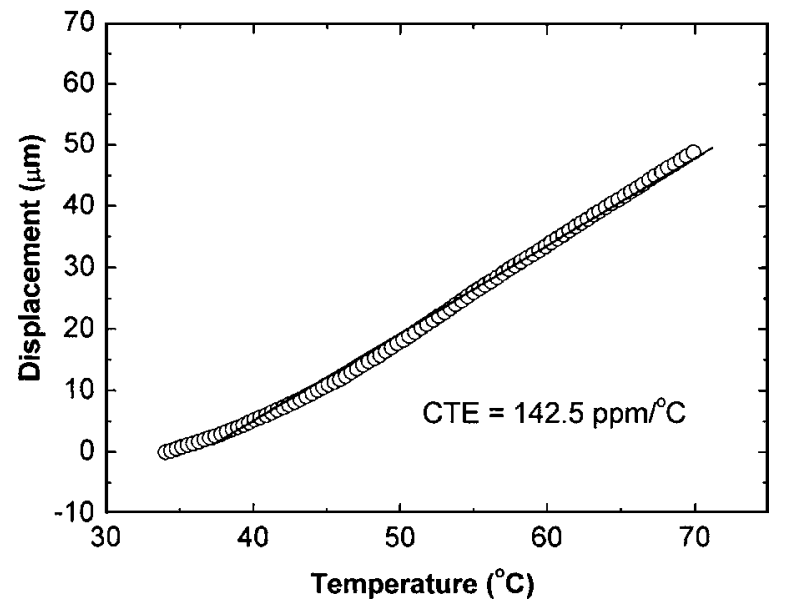

FIG. 4. Displacement changes of NOA71 material with temperature measured by TMA measurements.

order of magnitude when a device was fabricated on the NOA71 substrate replacing the $\mathrm{Si}$ wafer.

In summary, temperature-insensitive polymer wavelength filters with $\mathrm{Si}_{3} \mathrm{~N}_{4}$ gratings were fabricated on a polymer substrate. Polymer waveguides on polymer substrates were fabricated on top of the Si wafer with a water soluble PVA interlayer between the $\mathrm{Si}$ wafer and polymer waveguides. After the completion of the waveguides, the polymer waveguides are separated by dissolving the PVA layer from the substrate to form all polymer waveguides. The novel process provides a way to overcome the problem of the thermal instability of the polymer substrate when the polymer waveguides are directly fabricated on polymer substrates. UV-curable NOA61 was used as the core layer and NOA71 as the cladding and the substrate, respectively. The polymeric wavelength filter fabricated on a NOA71 substrate exhibits one order of magnitude lower Bragg wavelength shift with temperature than on the Si wafer in the temperature range of $25-70{ }^{\circ} \mathrm{C}$, which is consistent with the theoretical prediction.

The authors thank the KOSET through CRM for financial support of this work.

${ }^{1}$ M. C. Oh, H. J. Lee, M. H. Lee, J. H. Ahn, and S. G. Han, Appl. Phys. Lett. 72, 1559 (1998)

${ }^{2}$ J.-W. Kang, M.-J. Kim, J.-P. Kim, S.-J. Yoo, J.-S. Kim, D. Y. Kim, and J.-J. Kim, Appl. Phys. Lett. 82, 3823 (2003).

${ }^{3}$ Y. Kokobun, N. Funato, and M. Takizawa, IEEE Photonics Technol. Lett. 5, 1297 (1993).

${ }^{4}$ E.-S. Kang, W.-S. Kim, D.-J. Kim, and B.-S. Bae, IEEE Photonics Technol. Lett. 16, 2625 (2004).

${ }^{5}$ Y. Kokubun, M. Takizawa, and S. Taga, Electron. Lett. 30, 1223 (1994).

${ }^{6}$ N. Keil, H. H. Yao, and C. Zawaddzki, Appl. Phys. B 73, 619 (2001).

${ }^{7}$ Y. Kokubun, S. Yoneda, and H. Tanaka, Electron. Lett. 32, 1998 (1996).

${ }^{8}$ D. Bosc and N. Hurault, IEEE Photonics Technol. Lett. 10, 1751 (1998).

${ }^{9}$ D. Y. Kim, S. K. Tripathy, L. Li, and J. Kumar, Appl. Phys. Lett. 66, 1166 (1995).

${ }^{10}$ S.-H. Nam, J.-W. Kang, and J.-J. Kim (unpublished).

${ }^{11}$ S.-H. Nam, J.-W. Kang, and J.-J. Kim (unpublished). 